\title{
Non-cell autonomous inhibition of the Hedgehog response due to impaired cholesterol synthesis requires Ptch1/2 function
}

Carina Jägers and Henk Roelink

Department of Molecular and Cell Biology, University of California, Berkeley

\section{Keywords:}

Ptch1/2

Hh signaling

Cholesterol Synthesis

Smith-Lemli-Opitz syndrome

Lathosterolosis 


\begin{abstract}
Congenital birth defects due to mutations of enzymes involved cholesterol synthesis, like SmithLemli-Opitz syndrome (SLOS) and Lathosterolosis are characterized by an accumulation of cholesterol precursors. The phenotype of both SLOS and Lathosterolosis have similarities to syndromes associated with abnormal Sonic hedgehog (Shh) signaling, consistent with the observation that cholesterol precursors and their derivative can inhibit Shh signaling. Two types of multipass membrane proteins play central roles in Shh signal transduction, the putative Resistance, Nodulation and Division (RND) antiporters Patched (Ptch)1 and -2, and the G-protein coupled receptor Smoothened (Smo). Sterols have been suggested as cargo for Ptch1/2, while Smo activity can affected both positively and negatively by steroidal molecules. We demonstrate that embryonic stem cells with mutations in the 7-dehydroxycholesterol reductase (7Dhcr) or sterol-C5-desaturase $(S c 5 d)$ gene reduce the $\mathrm{Hh}$ response in adjacent wildtype cells when grown in mosaic organoids. This non-cell autonomous inhibitory activity of the mutant cells requires the presence of both Ptch1 and Ptch2. These observations support a model in which late cholesterol precursors that accumulate in cells lacking 7Dhcr are the cargo for Ptch1 and Ptch2 efflux activity and mediate the non-cell autonomous inhibition of Smo.
\end{abstract}




\section{Introduction}

Hedgehog $(\mathrm{Hh})$ signaling is critically important for embryonic development and patterning in most animals. Initial events in the transduction of the Hedgehog $(\mathrm{Hh})$ signal include binding of the ligand to the 12-transmembrane hedgehog receptor Patched (Ptch) which releases a Ptchmediated inhibition of the putative G-protein coupled receptor Smoothened (Smo) (Izzi et al., 2011; Marigo et al., 1996; Taipale et al., 2002). In the absence of Hh, Ptch inhibits Smo nonstoichiometrically (Taipale et al., 2002) and non-cell autonomously (Roberts et al., 2016) via a mechanism that remains poorly understood. Mammals have two Ptch homologs, Ptch1 and Ptch2 (Ptch1/2) that share overlapping functions (Alfaro et al., 2014; Lee et al., 2006; Roberts et al., 2016). Ptch1/2 are members of the Resistance-Nodulation Division (RND) family of antiporters that are present in all domains of life (Zhang et al., 2018). In bacteria, RND antiporters export their cargo in exchange for protons (Tseng et al., 1999) a function possibly conserved in mammals, although it is possible that Ptch1/2 can utilize other cation gradients (Myers et al., 2017). Bacterial RND transporters pump small lipophilic or amphiphilic molecules to the outside and eukaryotic RNDs plausibly have a similar function.

Although the precise nature of the cargo of Ptch1/2 antiporter activity remains unknown, several observations suggest that it is a steroidal Smoothened antagonist. The secretion of vitamin D3, a derivative of the cholesterol precursor 7-Dehydrocholesterol (7DHC), is Ptch1-dependent (Hausmann et al., 2009) and both vitamin D3 and 7DHC can repress Smo activity (Bijlsma et al., 2006; Linder et al., 2015). Several other known Smo modulators have a steroidal backbone, including the steroidal alkaloid cyclopamine (Incardona et al., 1998; Keeler, 1969; Sharpe et al., 2015). Sterols are derived from lanosterol, which is modified in a series of enzymatic reactions in the postsqualene pathway, culminating in the production of cholesterol or vitamin D (Bloch, 1965; Chojnacki and Dallner, 1988; Ernster and Dallner, 1995; Takeyama et al., 1997). Several congenital syndromes are caused by the loss of enzymes catalyzing the last steps in the cholesterol synthesis pathway (Figure 1A). Smith-Lemli-Opitz (SLO) is caused by mutations in the gene encoding 3 $\beta$-hydroxysterol $\Delta 7$-reductase (7DHCR) (Fitzky et al., 1998), which catalyzes the reduction of the 7 double bond in 7-Dehydrodesmosterol (Kandutch and Russell, 1960). 
Phenotypically, SLOS is characterized by a distinctive facial appearance, cleft palate, microcephaly, a small, upturned nose, micrognathia, and ptosis. Furthermore, limb malformations like postaxial polydactyly, syndactyly of the second and third toe, and proximally placed thumbs are observed (SMITH et al., 1964). This syndrome has overlap with the malformations caused by altered Shh signaling. Biochemically, elevated levels of 7DHC and its isomer, 8-Dehydrocholesterol (8DHC) and decreased cholesterol are found in serum and tissue of SLO patients as well as in a mouse model (Tint et al., 1994), and increased 7DHC levels are used for diagnosis of SLO (Abuelo et al., 1995).

Lathosterolosis is caused by mutations in the gene encoding the $3 \beta$-hydroxysteroid- $\Delta 5$ desaturase (SC5D) which desaturates lathosterol to 7DHC during cholesterol synthesis (BrunettiPierri et al., 2002). Symptoms of lathosterolosis have similarities with those observed in SLOS, including postaxial polydactyly, microcephaly, micrognathia, and toe syndactyly (Brunetti-Pierri et al., 2002). In a lathosterolosis mouse model, lathosterol levels are elevated 60 fold whereas levels of cholesterol were decreased similar to the SLOS mouse model (Krakowiak et al., 2003). Circulating maternal cholesterol can be transported to the fetus, making it unlikely that SLOS and lathosterolosis are predominantly caused by low fetal cholesterol, but instead it is plausible that the accumulation of late sterol precursors contributes to these birth defects, possibly by suppressing Hh signaling (Incardona et al., 1998).

Cell types in the developing neural tube arise in at stereotypic positions in response to Shh signaling. An effective in vitro model of the neural tube are spinal cord organoids (SCOs), which are differentiated aggregates of approximately $10^{4}$ embryonic stem cells (ESCs) (Wichterle et al., 2002). Mosaic SCOs can be generated by mixing cells with genotypically diverse cell lines in varying ratios. This approach allows us to address the non-cell autonomous effects caused by genetic alterations. Here, we provide evidence that loss of $7 D h c r$ or Sc5d and associated accumulation of late sterol precursors can inhibit Shh signaling both cell-autonomously and noncell autonomously even in cells with an intact cholesterol synthesis pathway. The increased noncell autonomous inhibition requires the presence of Ptch $1 / 2$ function in those cells that 
accumulate the cholesterol precursors. These observations are consistent with a model that late sterol precursors are the cargo of Ptch1/2 antiporter activity in its ability to inhibit Smo non-cell autonomously.

\section{Results}

The loss of 7-Dehydrocholesterol reductase and/or Lathosterol 5-desaturase changes distribution of sterols

We have shown before that Ptch1/2 activity can inhibit Smo non-cell autonomously, supporting the notion that Ptch1/2 functions in the distribution and secretion of a Smo inhibitor (Roberts et al., 2016). To assess if this inhibitor could be a late sterol precursor, we made $\mathrm{Shh}^{-1-; 7 D h c r-}$ cells and found that these cells have reduced amounts of cholesterol after three days of serum starvation as compared to the parental Sh/- cells (Figure 1B). To assess more global changes in the distribution sterols, we visualized the distribution of Filipin, a fluorescent stain of sterols (Blanchette-Mackie, 2000) used in the diagnosis of Niemann-Pick disease type C (Sokol et al., 1988). Similar to SLOS, the phenotype of lathosterolosis overlaps with that of congenital Shh malformations, raising the question if similar mechanisms impair Shh signal transduction. Besides

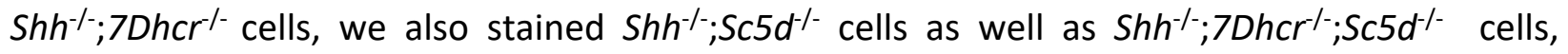
cultured as SCOs in the absence of serum for up to 72 hours. Shh/- cells had wrinkle-like accumulations of sterols in the plasma membrane on the first and second day of serum starvation (Figure 1C). However, few cells appeared with small rounder structures that accumulated on day 3. Cells lacking either 7Dhcr or Sc5d had an initial Filipin staining pattern similar to $S h^{-/-}$cells, while $\mathrm{Shh}^{-1-} ; 7 \mathrm{Dhcr}{ }^{-1-} ; \mathrm{Sc} \mathrm{d}^{-/-}$cells appeared smoother. At later timepoints, sterol distribution was different in $\mathrm{Shh}^{-1-} ; 7 \mathrm{DhCr}{ }^{-1-} ; \mathrm{Sc}^{-1 /-}$ cells with less sterol apparent on the plasma membrane and more pronounced staining in small domains, possibly endosomes. These results indicate that the Sc5d mutation is not strictly epistatic to the 7Dhcr mutation, as might be predicted as Sc5d and 7Dhcr are thought to act in sequence in the cholesterol synthesis pathway. The finding that these mutations have an additive effect might suggest that alternate, parallel pathways function in cholesterol synthesis. 


\section{Accumulation of late sterol precursors inhibits the Hh response}

The loss of 7Dher in SLOS is known to suppress Shh signaling, and a plausible hypothesis is that this is caused by the accumulation of a late sterol precursor that is inhibitory to Smo. To discern whether the accumulation of the cholesterol precursor 7DHC or the reduction of cholesterol can

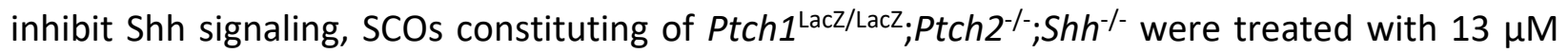
7DHC, $13 \mu \mathrm{M}$ cholesterol, or a combination of both sterols. Activation of Ptch1:LacZ, measured by LacZ activity, was used as an indicator for the Hh pathway response (Figure 1D) (Goodrich et al., 1997). The reporter cells lack of both Ptch1 and Ptch2, resulting in an activation of Smo and elevated Ptch1:LacZ expression levels compared to heterozygous reporter cells (Ptch1 ${ }^{+/ L a c Z}$;Shh/) (Alfaro et al., 2014; Roberts et al., 2016). Addition of 7DHC reduced the Shh pathway activity in Ptch1 ${ }^{\text {LacZ/LacZ;Ptch2 }}{ }^{-/-} ;$Sh $^{-/-}$SCOs by $30 \%$. Cholesterol had no effect on the Hh response, neither when added alone nor in combination with 7DHC. This demonstrates that under these conditions 7DHC is an inhibitor of the Hh response, while cholesterol has no effect and cannot antagonize the presence of 7DHC. These results show that the inhibitory cholesterol precursor can be transferred via the culture medium. Therefore, we tested the inhibitory activity of conditioned supernatants. We transferred medium of SCOs consisting of wild type, Ptch $1 / 2^{-/-}$or $7 D h c r^{-/}$cells

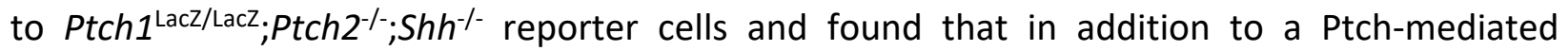
inhibition, the medium conditioned by $7 \mathrm{Dhcr}^{-/}$cells further decreased the $\mathrm{Hh}$ response in reporter cells by $40 \%$, but not medium conditioned by cells lacking Ptch1/2 activity (Figure 1E). These results support the idea that a cholesterol precursor enriched in the medium via Ptch1/2 activity can inhibit the Hh response non-cell autonomously.

\section{Loss of 7-Dehydrocholesterol reductase and lathosterol 5-desaturase inhibit the Shh pathway non-cell autonomously in a Ptch1/2-dependent manner}

To test if endogenous late cholesterol precursors like 7DHC or lathosterol can inhibit Shh signaling non-cell autonomously, we measured Ptch1:LacZ expression in Ptch1 ${ }^{\text {LacZ/LacZ;Ptch2 }}$-/-;Shh ${ }^{-/}$ reporter cells incorporated into mosaic SCOs that also contain $50 \% \mathrm{Shh}^{-1-} ; 7 \mathrm{Dhcr}^{-/}, \mathrm{Shh}^{-1-} ; \mathrm{Sc}^{-\mathrm{d}^{-/}}$or

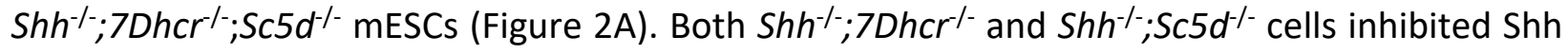
pathway activity in the neighboring reporter cells (Figure 2B). The inhibition by $\mathrm{Shh}^{-/-} ; \mathrm{DDhCr}^{-/}$ cells, however, was stronger than that by $S \mathrm{Sh}^{-/-} ; S c 5 d^{-/-} \mathrm{mESCs}$, suggesting that 7DHC is an 


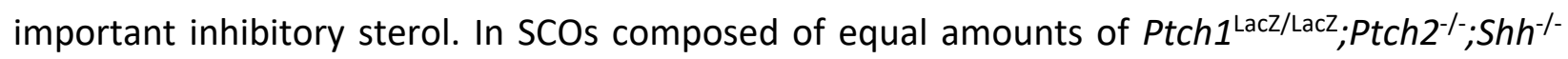

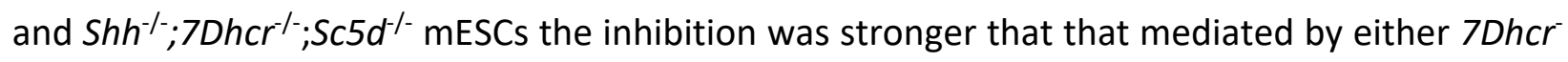
I- or $S c 5 d^{-/-}$cell alone. This additive effect is not easily reconciled with the idea of a linear pathway with 7Dhcr and Sc5d being enzymes acting in sequence but is consistent with and additive effect of these enzymes in sterol distribution (Figure 1C). The differences in the inhibitory potential of the different cell lines could not be explained by shifted ratios of reporter and repressor cells since the amount of CMFDA Green stained reporter cells remained the same in all 4 different types of mosaic $n E B s 48 \mathrm{~h}$ after aggregation (Figure $2 \mathrm{C}$ ). These results indicate that several early cholesterol precursors can inhibit Shh signaling non-cell autonomously. Moreover, the additive inhibitory effect of $\mathrm{Shh}^{-/-} ; 7 \mathrm{Dhcr}^{-/} ; \mathrm{Sc}_{5} \mathrm{~d}^{-/-}$on the Shh pathway in not readily consistent the canonical linear cholesterol biosynthesis pathway.

\section{Ptch1/2 activity is required for non-cell autonomous inhibition}

A central question that remains is if Ptch1/2 function is required of the non-cell autonomous inhibition of the Hh response by late sterol precursors. We compared the response to 7DHC in SCOs consisting of $100 \%$ Ptch1 $1^{\text {Lacz/LacZ }} ;$ Ptch $^{-/} ; \mathrm{Shh}^{-/}$cells to mosaic nEBs consisting of $50 \%$ Ptch1 ${ }^{\text {LacZ/LacZ;Ptch2 }}{ }^{-/-} ;$Sh $^{-/-}$reporter and 50\% Ptch1/2-containing cells (Figure 3A). Exogenously

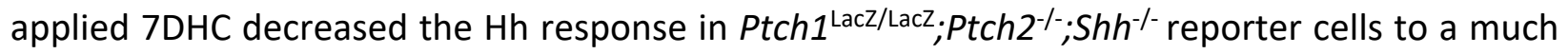
greater extend when Ptch1/2-containing cells were present in the same SCO, compounding the inhibitory function of Ptch1/2 alone. This suggests that 7DHC from the medium can be acted upon, and potentiated by Ptch1/2 function to inhibit the Hh response non-cell autonomously. Ptch1/2 function was unable to potentiate Smo antagonist cyclopamine, suggesting that cyclopamine inhibits Smo directly independent of Ptch1/2 function (Figure S1).

We tested if Ptch1/2 is sufficient for non-cell autonomous inhibition by cells mutant for 7Dhcr or Sc5d. We expressed Ptch1 or Ptch2 in Ptch1/2- or 7Dhcr-deficient fibroblasts (Ptch1 ${ }^{\text {Lacz/LacZ; Ptch2 }}$ /- and Ptch1 ${ }^{\text {LacZ/LacZ; }}$ Ptch2 ${ }^{-/-} ; 7 \mathrm{Dhcr}^{-{ }^{-}}$) and transferred their supernatant onto Light2 reporter cells. Conditioned medium from Ptch1- and Ptch2-expressing cells inhibited the Shh pathway response in the presence of SAG equally and independent of 7Dhcr in the conditioning cells (Figure 3B). 
To further assess the role of Ptch1/2 in non-cell autonomous inhibition of Shh signaling by cholesterol precursors, we mixed Ptch1/2-deficient Ptch1 Lacz/LacZ;Ptch2 $^{-/-} ;$Sh $^{-/-}$and

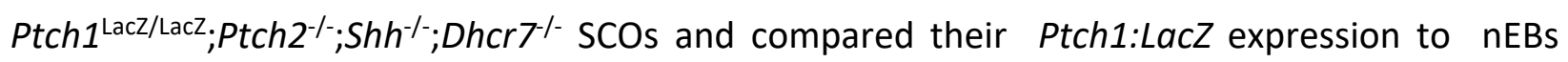
consisting of either genotype alone. As expected, the Hh response is constitutively high in

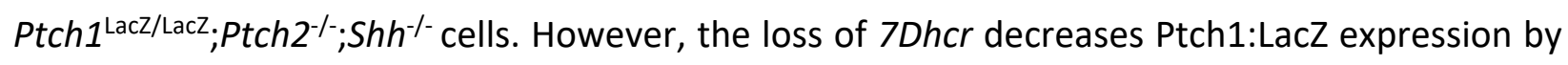
approximately $70 \%$ (Figure $3 \mathrm{C}$ ). If Ptch1/2 are indeed required for non-cell autonomous Shh pathway inhibition, 7Dhcr- and Ptch1/2 deficient cells should not be able to inhibit the Shh pathway response and the Ptch1:LacZ expression of the mosaic nEBs should be exactly half of

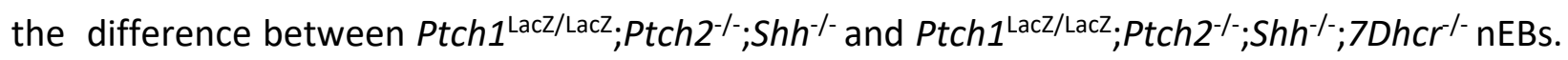
The null hypothesis would therefore be $\mathrm{H}_{0}=1-1 / 2$ (average Ptch $1^{\text {LacZ/LacZ; }}$ Ptch2 ${ }^{-/-} ;$Sh h $^{-/-}$- average

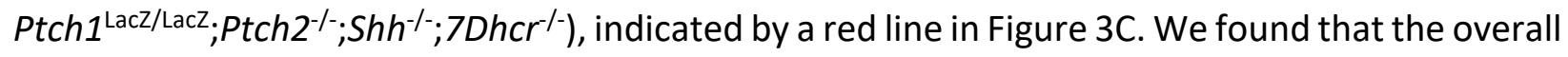
Hh response in Ptch1/2-deficient mosaic SCOs was nearly the same as $\mathrm{H}_{0}$, supporting the hypothesis that cells lacking Ptch1/2 function cannot inhibit the Hh response non-cell autonomously.

To further discern the role of Ptch1/2 in repressing and responding cells, we mixed 5\% Hb9:gfp mESCs (Wichterle et al., 2002) with a panel of Ptch1/2-proficient/deficient and/or 7Dhcrproficient/deficient mESCs. Hb9:gfp mESCs carry a transgene in which the Hh-sensitive $H b 9$ promoter drives $g f p$, and thus serve as reporters for activation of the Hh response (Figure 3D). The Hh pathway response was activated in the reporter cells with the Smo agonist SAG and the inhibitory potential of the interspersed cells was assessed by counting the number of Hb9:gfppositive nuclei as a measure of Hh pathway activation in SCOs (Wichterle et al., 2002). Compared to uninhibited Hb9:gfp reporter cells in Ptch1/2 -deficient mosaic nEBs, Ptch1/2 alone decreased the number of positive nuclei by more than half. Consistent with our results described earlier, Ptch1/2 containing but 7Dhcr deficient cells decreased the number of positive nuclei even further. In the absence of Ptch1/2 and 7Dhcr, however, Hb9:gfp expression was uninhibited, similar to the level of Ptch1/2-deficient mosaic nEBs. This demonstrates that a loss of Ptch1/2 activity is epistatic to a loss of 7Dhcr function in repressing cells and is consistent with the notion that Ptch $1 / 2$ is required for non-cell autonomous inhibition. As the addition of SAG increased the 
number of Hb9:gfp positive nuclei in every condition, the inhibitory factor in the $7 \mathrm{Dhcr} \mathrm{C}^{-/}$cells is possibly a competitive inhibitor to SAG, further indicating that Smo is the target of 7DHC inhibition of the $\mathrm{Hh}$ response (Figure 3E).

\section{Discussion}

In humans, mutations in the $D H C R 7$ gene that codes for $3 \beta$-hydroxysteroid $\Delta^{7}$-reductase are associated with the Smith-Lemli-Opitz Syndrome (SLOS), a congenital malformation syndrome (Fitzky et al., 1998). Some of the SLOS-associated malformations resemble birth defects observed in animals with reduced Hh signaling (Roessler et al., 1996), raising the hypothesis that the accumulating cholesterol precursor 7DHC reduces Shh signaling. Here, Shh ${ }^{-1-} ; 7 D h r^{-1-}$ mESCs were found to inhibit the Shh pathway non-cell autonomously. Importantly, Ptch1/2 was found to enhance the inhibitory effect of exogenously added 7DHC on reporter cells devoid of Ptch1/2, arguing for 7DHC being an endogenous cargo of Ptch1/2. Furthermore, Ptch1/2 are required for non-cell autonomous inhibition as 7Dchr-/- cells also deficient for Ptch1/2 were not able to inhibit the $\mathrm{Hh}$ response in adjacent cells.

\section{Possible scenario for the Ptch1/2-dependent inhibition of Smo by cholesterol precursors}

We propose the following model based on the data presented here (Figure 4): Ptch1/2 is able to inhibit Smo non-cell autonomously and its inhibitory function is even enhanced when the cells are deficient for one or more enzymes of the cholesterol synthesis pathway. This is likely due to the Ptch1/2-mediated transport of inhibitory cholesterol precursors like 7DHC, increasing their inhibitory potential by a yet unknown mechanism (Bijlsma et al., 2006; Linder et al., 2015). To this point, we cannot exclude that other sterols that accumulate in the absence of 7Dhcr or Sc5d are the preferred cargo of Ptch1/2 that inhibits Smo.

\section{Does Ptch transport a Smo-inhibitory sterol?}

There are many Smo-modulating small molecules, both endogenous and exogenous. Several of the molecules affecting Smo activity have a steroidal structure. Together with evidence that Ptch1/2 can inhibit Smo non-cell autonomously, suggests that in the absence of the Hh ligand, Ptch transports a sterol that inhibits Smo. Cyclopamine, a steroidal alkaloid inhibits Smo via direct binding (Chen et al., 2002). However, we find that Ptch1/2 does not exacerbate Smo inhibition 
by cyclopamine (Figure S1), arguing against that this steroidal alkaloid is a cargo of Ptch1/2. The ubiquity of cholesterol makes it an unlikely substrate for the Ptch1/2 function in Smo inhibition. However, late cholesterol precursors are good candidates. Vitamin D3 is derived from 7DHC via photolysis (Holick and Clark, 1978). When 7DHC accumulates in the absence of 7Dhcr, the synthesis of vitamin D3 is increased (Prabhu et al., 2016). Vitamin D3/cholecalciferol, the precursor of vitamin D3, is capable of inhibiting Smo more efficiently than 7DHC and is secreted by Ptch1 (Bijlsma et al., 2006). Furthermore, vitamin D3, was reported to be released into the extracellular space by Ptch1 and efficiently inhibit the translocation of Smo to the primary cilium (Linder et al., 2015).

\section{Evidence for alternative pathways in the cholesterol biosynthesis pathway}

Cholesterol synthesis begins with acetyl-CoA and has a multiplicity of intermediates, each requiring catalyzation by unique enzymes to progress to the next precursor molecule. Kandutsch and Russell proposed a linear but bifurcated pathway in 1960 for the synthesis of cholesterol that only differ in an additional double bond in the alkyl side chain appended to the CD-ring. This double bond can be resolved at any time by the $3 \beta$-hydroxysterol $\Delta^{24}$-reductase. Sc5d acts upstream of 7Dhcr and a mutation would therefore be predicted to be epistatic to a mutation in the 7Dhor gene. Contrary, we observed an additive inhibitory effect of the two mutations in combination on the Shh pathway. Thus, additional functions of these enzymes or additional, parallel pathways in the final steps of cholesterol synthesis are conceivable. Porter et. al proposed, with reservation, that 8-Dehydrocholesterol (8DHC), an isomer of 7DHC, can be synthesized from the precursor of lathosterol (cholesta-8(9)-en-3 $\beta$-ol) by Sc5d (Porter, 2002; Porter and Herman, 2011). 8DHC and 7DHC differ in the position of a double bond that can be swapped in both directions by the enzyme that synthesizes lathosterol from its precursor, $3 \beta$ hydroxysterol $\Delta 8, \Delta 7$-isomerase (EBP). Although this alternative pathway does not explain the additive inhibitory effect of combinatorial Sc5d and 7Dhcr knock-outs - a lack of Sc5d would still terminate the synthesis of cholesterol at the level of lathosterol - it might suggest that the cholesterol synthesis pathway is not strictly linear and that enzymes of cholesterol synthesis can exhibit additional functions. 


\section{The mechanism of Smo inhibition by Ptch1/2}

The hypothesis that the non-cell autonomous inhibition of the Shh pathway by Ptch $1 / 2$ is mediated by its antiporter function originates from the observation that a mutation in the antiporter channel (Ptch1D499A) blocks Ptch1 activity and subsequently, the Hh response (Alfaro et al., 2014; Taipale et al., 2002). Further evidence of this model came from the observation that the Ptch1/2-mediated inhibition of Smo is non-cell autonomous (Roberts et al., 2016).

As a member of the RND family of proton driven antiporters Ptch1/2 is thought to require a proton gradient to transport molecules across membranes (Tseng et al., 1999). Sodium has been proposed as an alternative ion that would be transported across the cell membrane in exchange for the Smo inhibitory molecule. Besides the question of "what" Ptch1/2 transports, the "how/where" has been the point of more recent discussion. The Ptch1 monomer revealed a hydrophobic pore-like structure in cryo-electron microscopy (cryo-EM) models that can be occupied by the N-terminus of Shh. In bacterial RNDs this is the channel that lets in protons and blocking this channel by Shh would provide a simple mechanism by which Shh blocks Ptch1/2 function. Dispatched, another member of the RND superfamily, is a homotrimer (Etheridge et al., 2010), as is Drosophila Ptch (Lu et al., 2006) indicating that also Ptch1 functions as a trimer. In bacterial RNDs trimerization forms a central channel through which secreted cargo leaves the cell, and that Ptch would function like bacterial RNDs, something indicated by conservation in their structures.

\section{Materials and Methods}

\section{CRISPR/Cas9 mediated mutagenesis}

The CRISPR/Cas9 genome editing technique was used to knock out the 7Dhcr and Sc5d gene (Ran et al. 2013). The pX458/ pSpCas9(BB)-2A-Puro and pX459/ pSpCas9(BB)-2A-GFP expression plasmids were a gift from the Doudna lab (University of California, Berkeley, USA). sgRNAs were designed using the online tool provided by the Zhang lab (http://tools.genome-engineering.org, MIT, Massachusetts, USA) and ordered from IDT DNA Technologies (lowa, USA). Cloning of sgRNA and Cas9 expressing plasmids was performed according to (Ran et al., 2013) 


\section{Tissue culture}

Ptch1 ${ }^{\text {Lacz/LacZ; }}$ Ptch2 $2^{-/-} ;$Sh $^{-/-}$and Shh ${ }^{-/-}$mESCs were generated using TALEN mediated mutagenesis

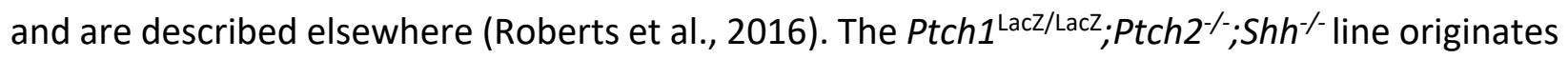
from the Ptch1 $1^{\text {Lacz/LacZ }}$ line (Goodrich et al., 1997). All cell lines were cultured under standard conditions in ES medium (DMEM/Gibco, 15 \% FBS/Gibco, 2mM L-glutamine/Thermo Fisher Scientific, 1X Penicillin Streptomycin/Thermo Fisher Scientific, 1X MEM Non-Essential Amino Acids Solution/Thermo Fisher Scientific, 1X Nucleosides for ES cells/EMD Millipore, 1X ßMercaptoethanol, 1X GST-LIF/Millipore) in tissue culture dishes coated with gelatin.

For generation of SCOs, $2 \times 10^{5}-4 \times 10^{5}$ mESCs were cultured in 60mm Petri dishes in DFNB medium (Neurobasal medium/Gibco, DMEM F12 1:1/Gibco, 1.5 mM L-glutamine/Thermo Fisher Scientific, 1X Penicillin Streptomycin/Thermo Fisher Scientific, 1X B27 ${ }^{\circledR}$ Supplement/Gibco, 0.1 $\mathrm{mM}$ ß-Mercaptoethanol) on a rotating platform. After 48 h, $10 \mu \mathrm{M}$ Retinoic Acid (Sigma/Aldrich) was added.

7DHC, cholesterol, and lathosterol (all Sigma/Aldrich) were dissolved in ethanol and used at 13 $\mu \mathrm{M}$. All reagents were added directly to aggregating SCOs.

Fibroblasts were grown in DMEM $+10 \%$ FBS (Atlas Biologicals) and transfected with Lipofectamine2000 according to the manufacturer's instructions. The medium was exchanged with DMEM $24 \mathrm{~h}$ after transfection for medium conditioning (24h). The conditioned medium was then removed, filtered, and transferred onto Light2 cells for another 48h with $30 \mathrm{nM} \mathrm{SAG.} \mathrm{Gli-}$ and Renilla-luciferase levels in the Light2 cells were measured with the Dual luciferase kit according to the manufacturer's instructions. Gli luciferase/Renilla luciferase levels were normalized to the respective mock-transfected control.

\section{Cell tracking}

For cell lineage tracking experiments, cells were incubated with $500 \mu \mathrm{M}$ CMFDA Green or CMAC Blue (both Invitrogen) in DFNB medium for $45 \mathrm{~min}$ at $37^{\circ} \mathrm{C}$ and seeded as described above. After $48 \mathrm{~h}$ (or respective time points), SCOs were fixed in $4 \%$ paraformaldehyde (Thermo Fisher Scientific) in $1 \mathrm{X}$ PBS for $10 \mathrm{~min}$ and mounted directly in Fluoromount G Mounting Medium 
(Thermo Fisher Scientific). Images were taken with a Zeiss Observer fluorescence microscope with a $20 \mathrm{X}$ and $10 \mathrm{X}$ objective.

\section{Filipin staining}

For filipin staining, SCOs were fixed in $4 \%$ paraformaldehyde (Thermo Fisher Scientific) in 1X PBS for 10 min after one, two, or three days. SCOs were stained in $30 \mu \mathrm{M}$ filipin III (Sigma/Aldrich) in 1X PBS for 30 min in the dark and mounted in Fluoromount G Mounting Medium (Thermo Fisher Scientific) after washing once with 1X PBS. Images were taken with a Zeiss Observer fluorescence microscope with a 63X objective and oil immersion.

\section{Reporter Gene Assay for Ptch1:LacZ induction}

Ptch1:LacZ expression levels were quantified $72 \mathrm{~h}$ after aggregation of SCOS using the GalactoLight Plus ${ }^{\mathrm{TM}}$ system (Applied Biosciences) according to the manufacturer's instructions. Shortly, SCOs were collected in plastic tubes, washed with 1X PBS, and lysed using lysis buffer (100 mM potassium phosphate $\mathrm{pH} 7.8,0.2 \%$ Triton X-100). Lysates were incubated with $70 \mu$ l Reaction buffer for $30 \mathrm{~min}$ in a 96 well plate, followed by an incubation with Accelerator-II for $15 \mathrm{~min}$. Signal was read in a microplate luminometer for $5 \mathrm{~s}$ per well. Ptch1:LacZ levels were normalized to total protein using the Bradford reagent (BioRad).

\section{Statistics}

Single Factor ANOVA was used to analyze more than two conditions, followed by a Student's $t$ test with a two-tailed distribution assuming unequal variance comparing two conditions. $* p<0.05, * * p<0.01, * * * p<0.001, * * * * p<0.0001$

\section{Funding.}

This work was supported by NIH grant R01GM117090 to HR.

\section{Acknowledgements.}

We thank Dr. Anthony T. Lavarone at the QB3/Chemistry Mass Spectrometry Facility (UC 
bioRxiv preprint doi: https://doi.org/10.1101/2020.12.10.420588; this version posted February 9, 2021. The copyright holder for this preprint (which was not certified by peer review) is the author/funder, who has granted bioRxiv a license to display the preprint in perpetuity. It is made available under aCC-BY-NC-ND 4.0 International license.

Berkeley) for help with obtaining the mass spectrometry data.

\section{Author contributions.}

All experiments were performed by CJ. Experiments were designed by $\mathrm{CJ}$ and HR. The manuscript was written by $\mathrm{CJ}$ and $\mathrm{HR}$.

\section{Conflict of interest.}

The authors declare no conflict of interest. 
A

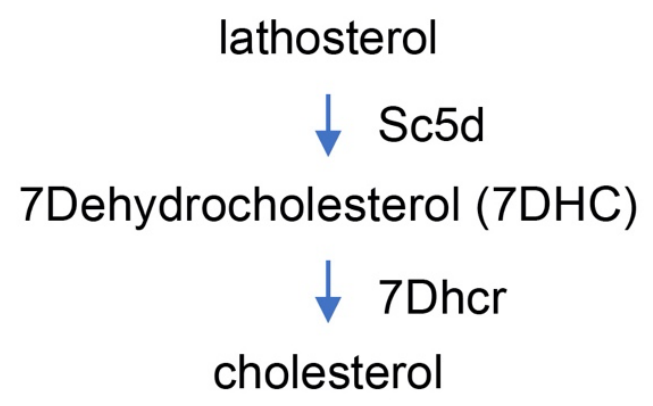

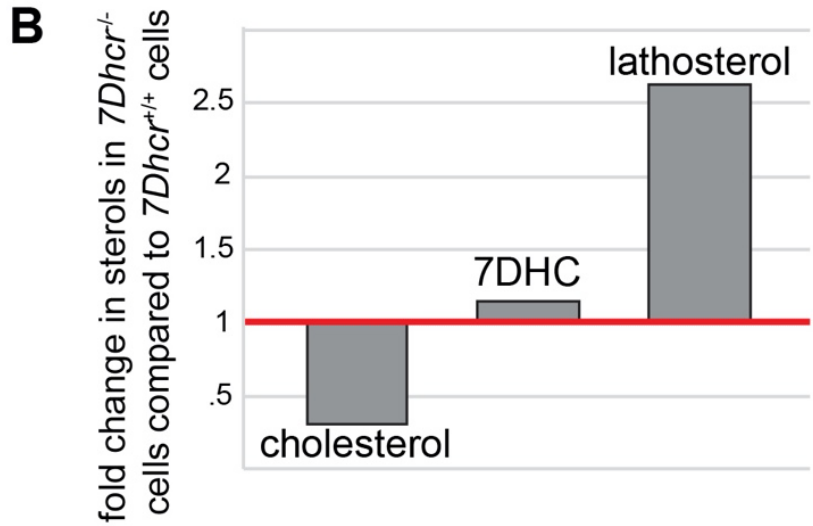
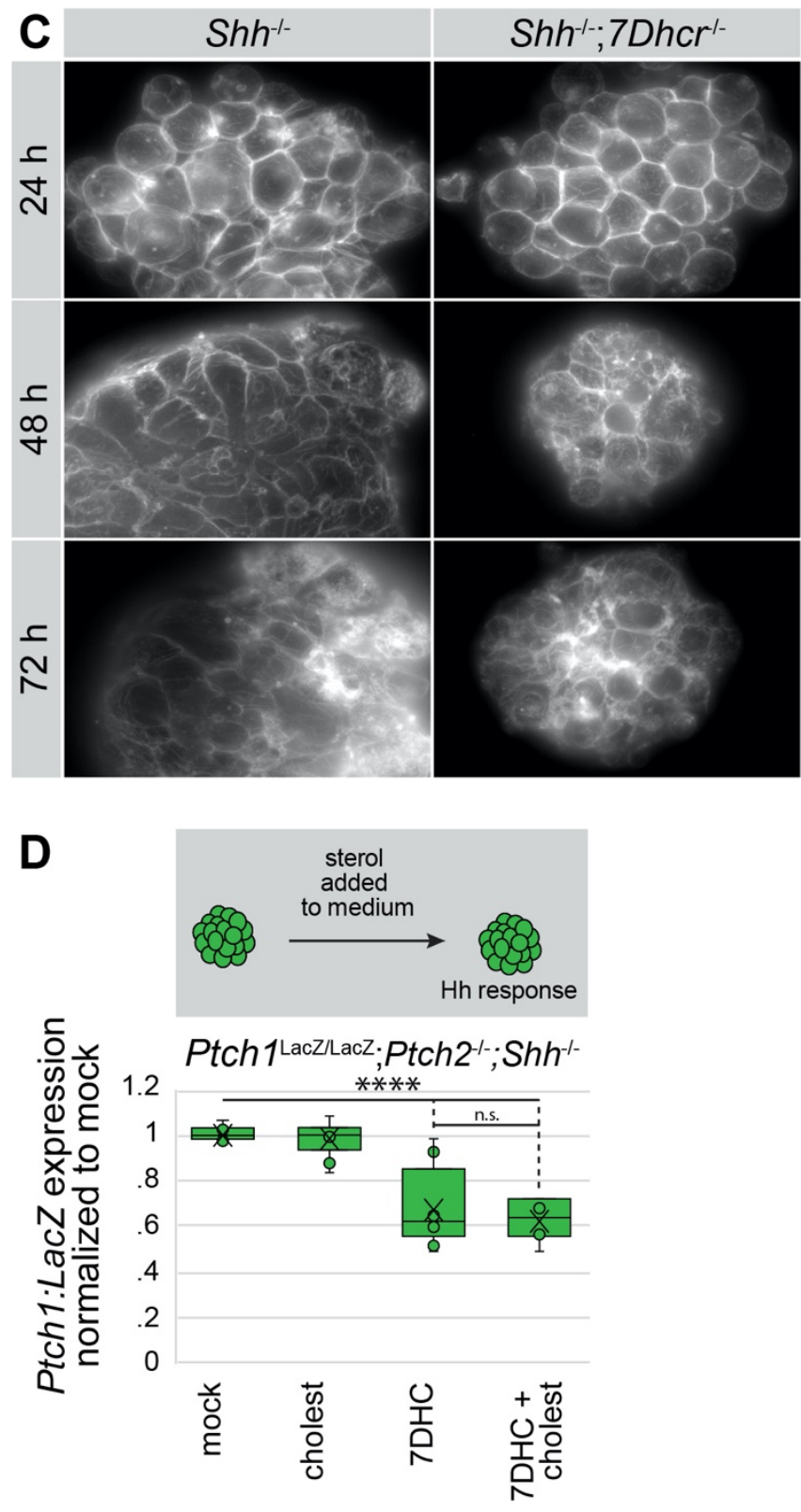

E
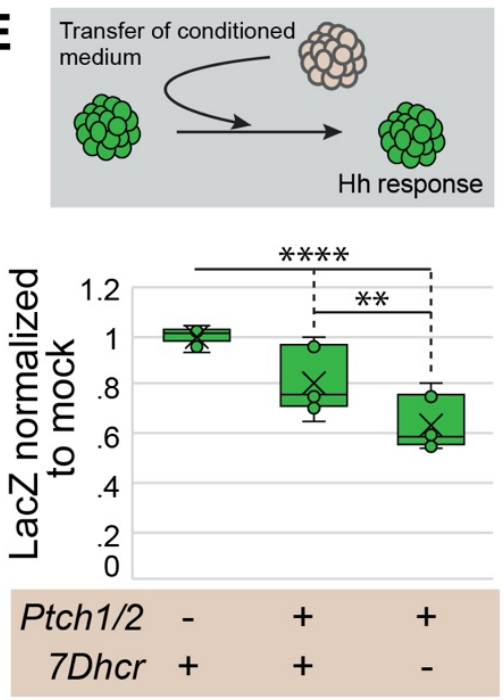


\section{Figure 1: $\mathrm{Shh}^{-/-} ; 7 \mathrm{Dhcr}{ }^{-/}$inhibit the Hh response non-cell autonomously}

A: Schematic of the last enzymatic reactions in cholesterol synthesis. Lathosterol is converted to 7DHC by the desaturase Sc5d, and 7DHC to cholesterol by the desaturase 7Dhcr. B: Cholesterol, 7DHC, and lathosterol levels were measured in $7 \mathrm{Dhcr}^{-/}$cells 3 days after serum starvation by gas chromatography mass spectrometry (GC-MS) and compared to $7 \mathrm{Dhcr}^{+/+}$cells. C: Staining of

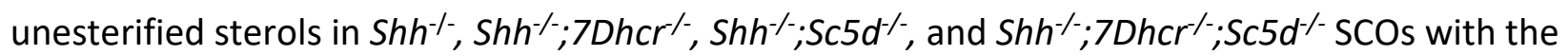
fluorescent dye Filipin 3 days after serum starvation. D: Ptch1:LacZ measurement of Ptch1 ${ }^{\text {LacZ/LacZ; }}$ Ptch2 ${ }^{-/} ;$Sh $^{-/}$reporter cells in SCOs that were treated with mock, $13 \mu \mathrm{M}$ 7DHC, 13 $\mu \mathrm{M}$ cholesterol, or $13 \mu \mathrm{M} 7 \mathrm{DHC}$ and $13 \mu \mathrm{M}$ cholesterol for 3 days under serum starvation. Box-

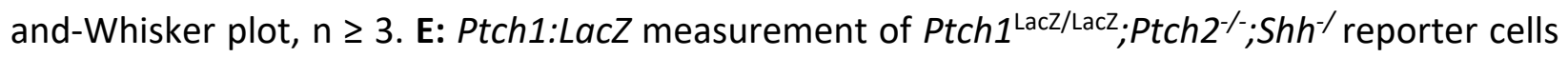
in SCOs that were cultured in medium conditioned by Ptch1 ${ }^{\mathrm{LacZ} / \mathrm{LacZ}} ; \mathrm{Ptch}^{-\%} ; \mathrm{Shh}^{\%}$, $\mathrm{Shh}^{-/}$, or Shh ${ }^{-/}$ ;7Dhcr/- SCOs. Box-and-Whisker plot, $\mathrm{n}=3$. $* * * * p<0.0001, * * p<0.01$, n.s. not significant. 
A
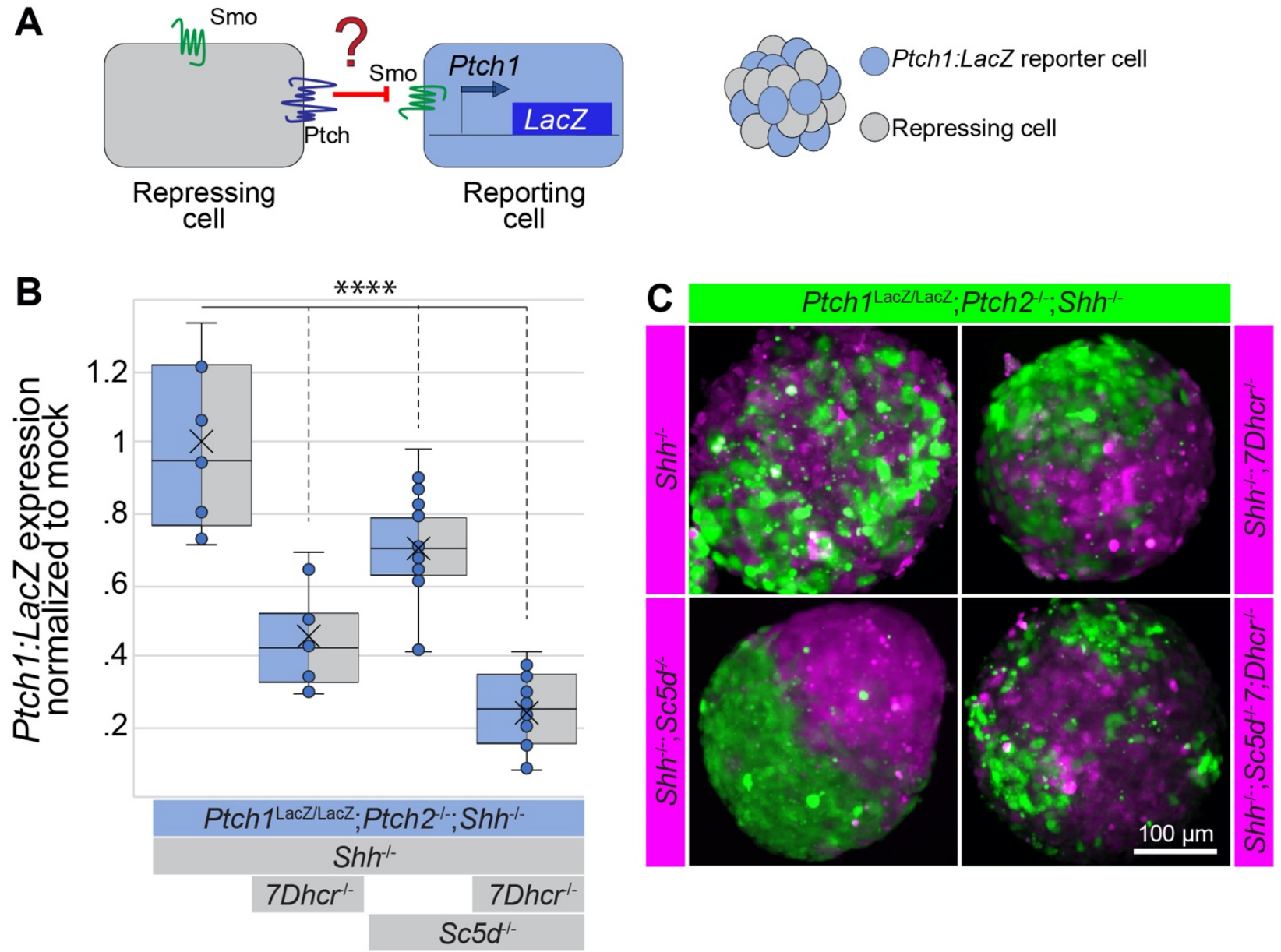

Figure 2: Non-cell autonomous inhibition of the Hh response by cells mutant for enzymes of cholesterol synthesis.

A: Experimental setup: In mosaic SCOs consisting of equal amounts of reporter and repressor cells, repressor cells have Ptch1/2 but lack varying enzymes of cholesterol synthesis. The reporter cells with a Ptch1:LacZ reporter gene lack Ptch1/2 and therefore have an upregulated

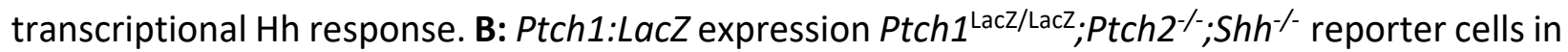
mosaic SCOs after 3 days of serum starvation. Box-and-Whisker plot, $\mathrm{n}=5, * * * * p<0.0001$. C: Fluorescent images of mosaic SCOs consisting of reporter and repressor cells (genotypes

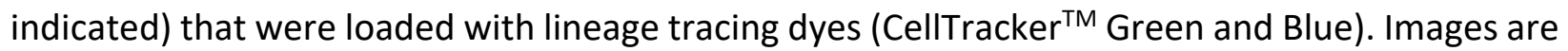
pseudo-colored. 


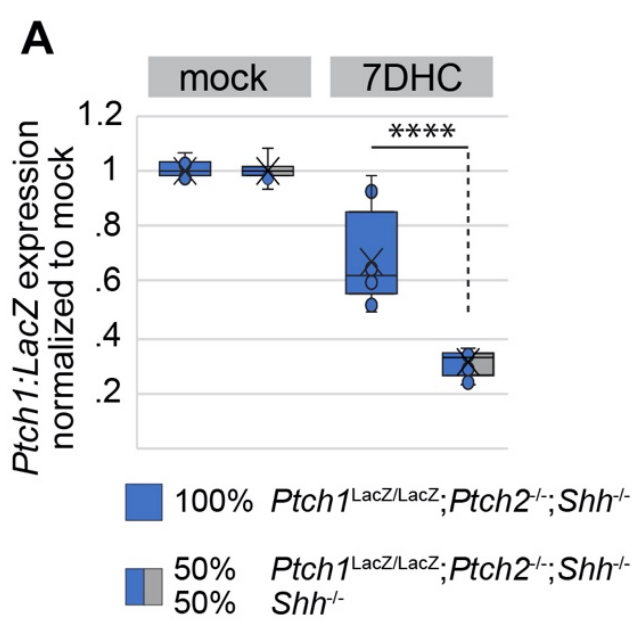

\section{Hh response:}

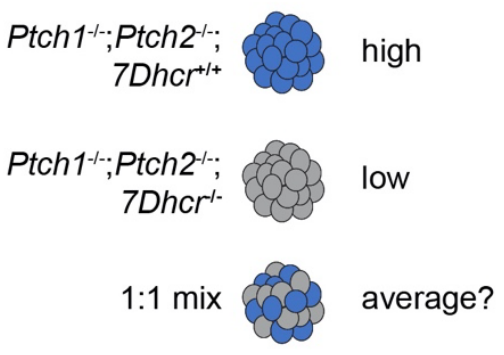

Null hypothesis $\left(\mathrm{H}_{0}\right)$ : the $\mathrm{Hh}$ response in mosaic organoids with $50 \% 7 \mathrm{Dhcr} /-$ and $50 \% 7 \mathrm{Dhcr}^{+1+}$ cells will be the average of the individual organoids

\section{Hbg:gfp reporter cell \\ Repressor cell}

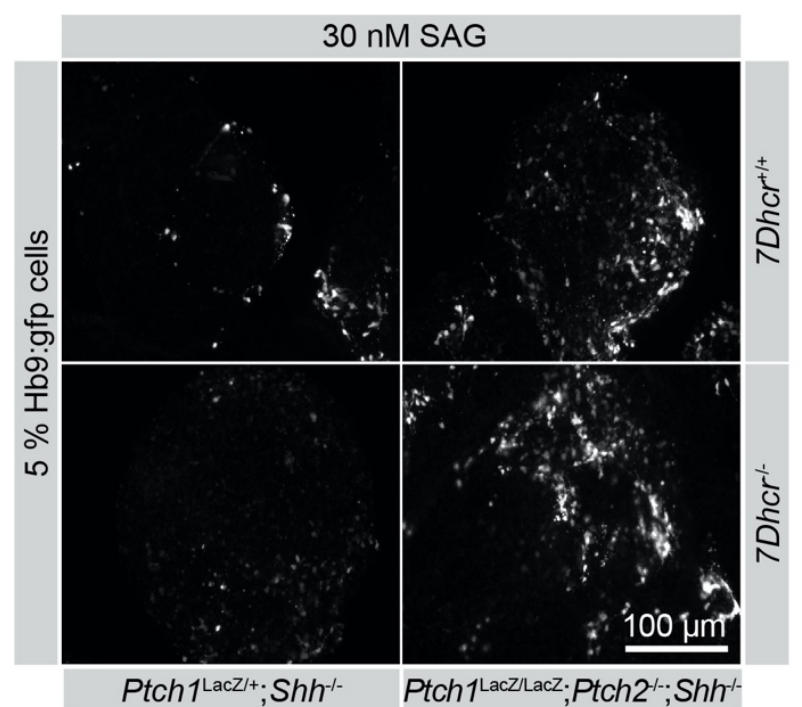

B

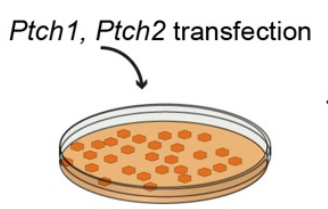

medium transfer

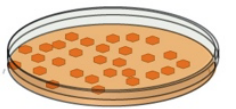

Light2 cells
Conditioned medium from

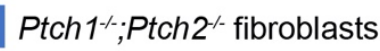

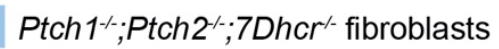

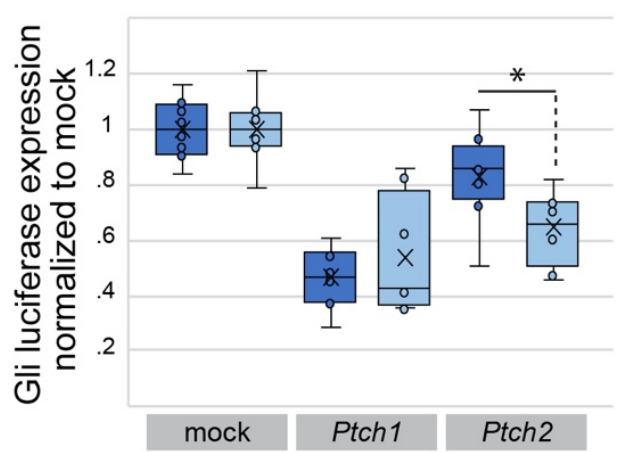

E

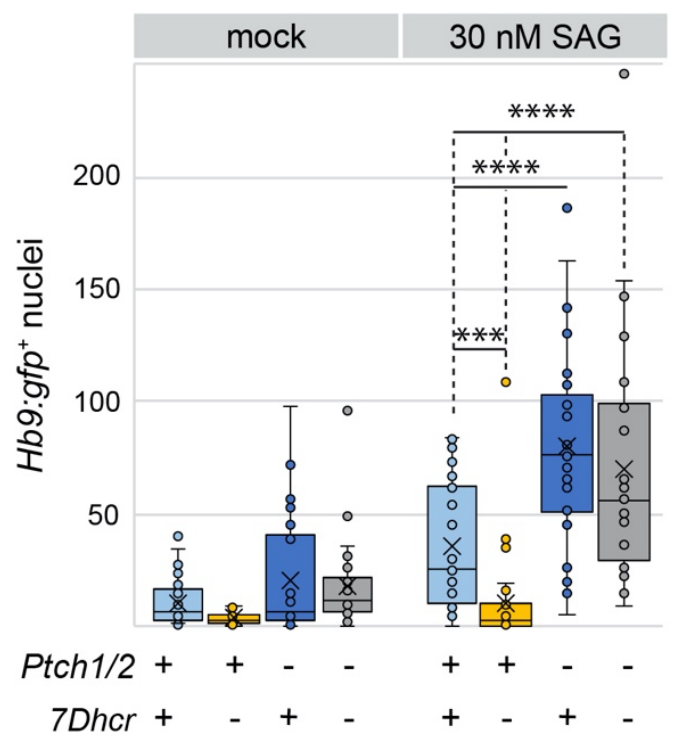


Figure 3: Non-cell autonomous inhibition by late cholesterol precursors requires Ptch1/2.

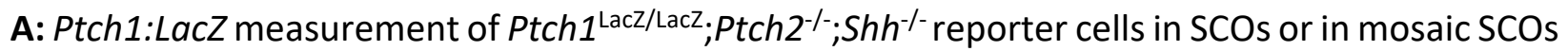
with $50 \% \mathrm{Shh}^{-/} \mathrm{mESC}$ after 3 days of serum starvation. $13 \mu \mathrm{M}$ 7DHC was added after the first night of SCO aggregation. Ptch1:LacZ levels are normalized to the respective mock treatment to account for lower numbers of reporter cells and inhibition by Ptch1/2 in mosaic SCOs. Box-andWhisker plot, $\mathrm{n}=4$. B: Experimental setup and Gli luciferase measurements of $\mathrm{Hh}$ responsive

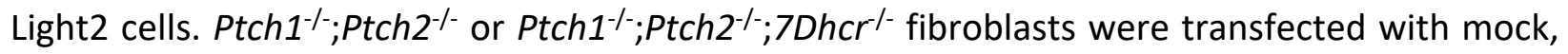
Ptch1, or Ptch2 and their conditioned medium was filtered and transferred onto Light2 cells with 30 nM SAG. Gli luciferase levels were normalized to Renilla luciferase and all conditions to their respective mock transfection. Box-and-Whisker plot, $n=4$. C: Graphic representation of the null hypothesis (indicated by red line): If Ptch1/2 is not required for non-cell autonomous inhibition, Ptch1:LacZ expression in mosaic SCOs will be the average of the individual SCOs. Ptch1:LacZ

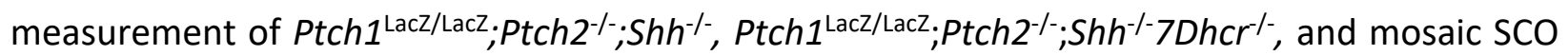
consisting of equal amounts of the two reporter lines 3 days after serum starvation. Box-andWhisker plot, $\mathrm{n}=4$. D: Mosaic SCOs with 5\% Hb9:gfp reporter cells and the indicated repressor cells were serum starved for 3 days and treated with 30 nM SAG for the last 24 hours. SCOs were fixed and Hb9:gfp expression imaged. Shown are representative images of mosaic SCOs for each condition. E: Quantification of Hb9:gfp positive nuclei of the experiment shown in D, shown in a Box-and-Whisker plot, $\mathrm{n}=3$. $^{*} p<0.05,{ }^{* * *} p<0.001, * * * * 00.0001$ 


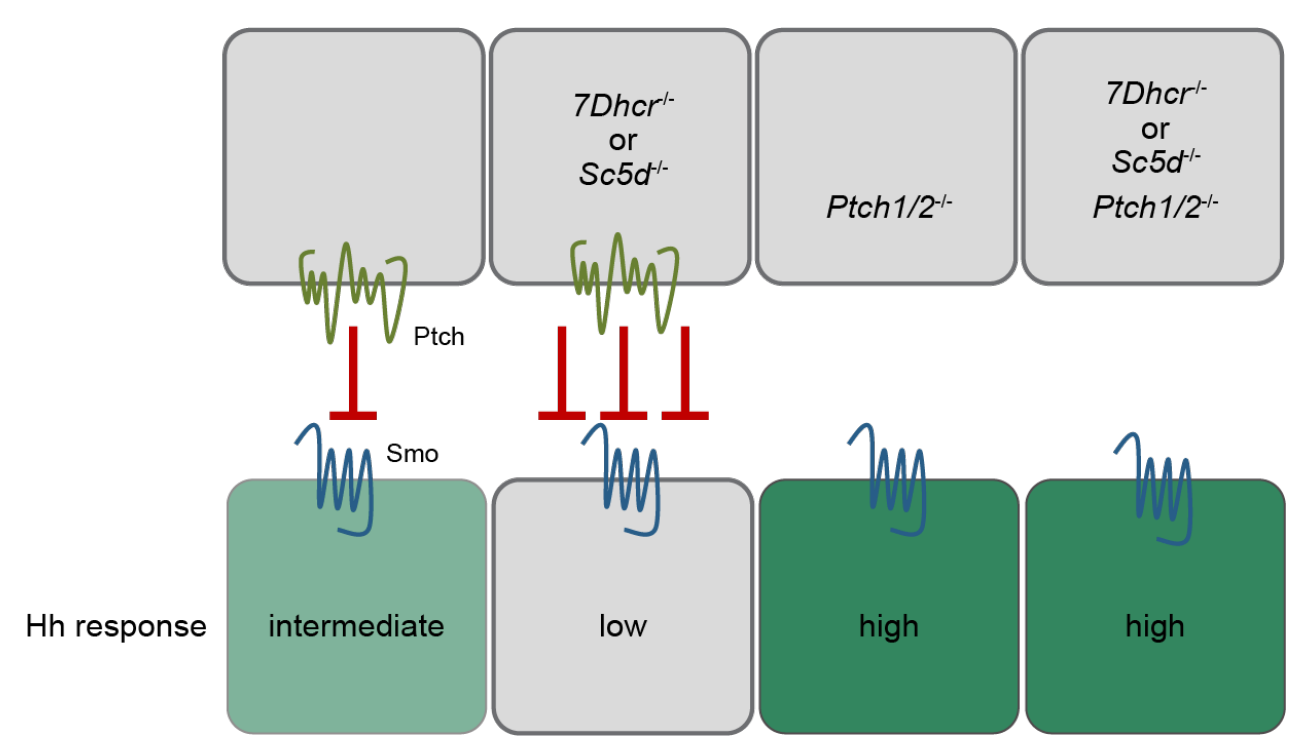

Figure 4: Model of non-cell autonomous Smo inhibition by Ptch1/2.

Ptch1/2 inhibits Smo non-cell autonomously and does so more potently when the Ptch1/2containing cells lack enzymes of cholesterol synthesis, resulting in a decreased transcriptional $\mathrm{Hh}$ response. In the absence of Ptch1/2 and independent of the absence or presence of enzymes of cholesterol synthesis, Smo in uninhibited and the transcriptional Hh response is high. 
A

$$
\text { cyclopamine }
$$
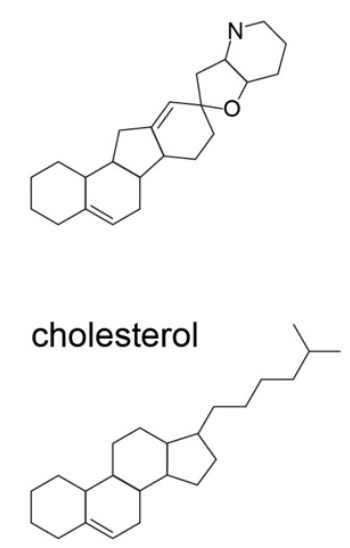

B

cyclopamine

mock

$10 \mathrm{nM}$

$30 \mathrm{nM}$

$100 \mathrm{nM}$

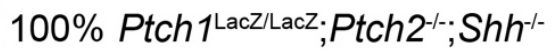

50\% Ptch1 LacZ/LacZ;Ptch2 ${ }^{-/-} ;$Sh $^{-1-}$

$50 \% \mathrm{Shh}^{-1-}$

Figure S1: Ptch1/2 does not enhance the inhibition of Smo by cyclopamine.

A: Structural comparison of the steroidal molecules cyclopamine and cholesterol. B: Ptch1:LacZ

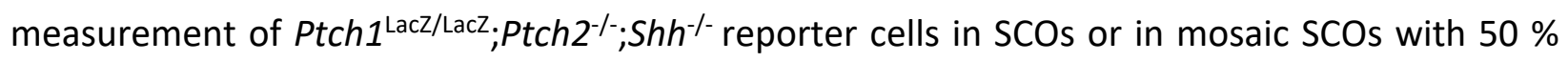
$\mathrm{Shh}^{-/-}$mESCs after 3 days of serum starvation. The indicated concentrations of cyclopamine were added after the first night of SCO aggregation. Ptch1:LacZ levels are normalized to the respective mock treatment to account for lower numbers of reporter cells and inhibition by Ptch $1 / 2$ in mosaic SCOs. Box-and-Whisker plot, $n=6, * p<0.05$. 


\section{References}

Abuelo, D. N., Tint, G. S., Kelley, R., Batta, A. K., Shefer, S. and Salen, G. (1995). Prenatal detection of the cholesterol biosynthetic defect in the Smith-Lemli-Opitz syndrome by the analysis of amniotic fluid sterols. American journal of medical genetics 56, 281-285.

Alfaro, A. C., Roberts, B., Kwong, L., Bijlsma, M. F. and Roelink, H. (2014). Ptch2 mediates the Shh response in Ptch1-/- cells. Development (Cambridge, England) 141, 3331-3339.

Bijlsma, M. F., Spek, C. A., Zivkovic, D., van de Water, S., Rezaee, F. and Peppelenbosch, M. P. (2006). Repression of smoothened by patched-dependent (pro-)vitamin D3 secretion. PLoS biology 4, e232.

Blanchette-Mackie, E. J. (2000). Intracellular cholesterol trafficking: role of the NPC1 protein. Biochimica et biophysica acta 1486, 171-183.

Bloch, K. (1965). The biological synthesis of cholesterol. Science (New York, N.Y 150, 19-28.

Brunetti-Pierri, N., Corso, G., Rossi, M., Ferrari, P., Balli, F., Rivasi, F., Annunziata, I., Ballabio, A., Russo, A. D., Andria, G., et al. (2002). Lathosterolosis, a novel multiplemalformation/mental retardation syndrome due to deficiency of 3beta-hydroxysteroiddelta5-desaturase. Am. J. Hum. Genet. 71, 952-958.

Chen, J. K., Taipale, J., Cooper, M. K. and Beachy, P. A. (2002). Inhibition of Hedgehog signaling by direct binding of cyclopamine to Smoothened. Genes \& development 16, 2743-2748.

Chojnacki, T. and Dallner, G. (1988). The biological role of dolichol. The Biochemical journal 251, 1-9.

Ernster, L. and Dallner, G. (1995). Biochemical, physiological and medical aspects of ubiquinone function. Biochimica et biophysica acta 1271, 195-204.

Etheridge, L. A., Crawford, T. Q., Zhang, S. and Roelink, H. (2010). Evidence for a role of vertebrate Disp1 in long-range Shh signaling. Development (Cambridge, England) 137, 133140.

Fitzky, B. U., Witsch Baumgartner, M., Erdel, M., Lee, J. N., Paik, Y. K., Glossmann, H., Utermann, G. and Moebius, F. F. (1998). Mutations in the Delta7-sterol reductase gene in patients with the Smith-Lemli-Opitz syndrome. Proceedings of the National Academy of Sciences of the United States of America 95, 8181-8186.

Goodrich, L. V., Milenkovic, L., Higgins, K. M. and Scott, M. P. (1997). Altered neural cell fates and medulloblastoma in mouse patched mutants. Science (New York, N.Y 277, 1109-1113. 
Hausmann, G., Mering, von, C. and Basler, K. (2009). The hedgehog signaling pathway: where did it come from? PLoS biology 7, e1000146.

Holick, M. F. and Clark, M. B. (1978). The photobiogenesis and metabolism of vitamin D. Federation proceedings 37, 2567-2574.

Incardona, J. P., Gaffield, W., Kapur, R. P. and Roelink, H. (1998). The teratogenic Veratrum alkaloid cyclopamine inhibits sonic hedgehog signal transduction. Development (Cambridge, England) 125, 3553-3562.

Izzi, L., Lévesque, M., Morin, S., Laniel, D., Wilkes, B. C., Mille, F., Krauss, R. S., McMahon, A. P., Allen, B. L. and Charron, F. (2011). Boc and Gas1 each form distinct Shh receptor complexes with Ptch1 and are required for Shh-mediated cell proliferation. Developmental cell 20, 788-801.

KANDUTSCH, A. A. and RUSSELL, A. E. (1960). Preputial gland tumor sterols. 3. A metabolic pathway from lanosterol to cholesterol. J. Biol. Chem. 235, 2256-2261.

Keeler, R. F. (1969). Teratogenic compounds of Veratrum californicum (Durand). VI. The structure of cyclopamine. Phytochemistry 8, 223-225.

Krakowiak, P. A., Wassif, C. A., Kratz, L., Cozma, D., Kovárová, M., Harris, G., Grinberg, A., Yang, Y., Hunter, A. G. W., Tsokos, M., et al. (2003). Lathosterolosis: an inborn error of human and murine cholesterol synthesis due to lathosterol 5-desaturase deficiency. Hum. Mol. Genet. 12, 1631-1641.

Lee, Y., Miller, H. L., Russell, H. R., Boyd, K., Curran, T. and McKinnon, P. J. (2006). Patched2 modulates tumorigenesis in patched1 heterozygous mice. Cancer research 66, 6964-6971.

Linder, B., Weber, S., Dittmann, K., Adamski, J., Hahn, H. and Uhmann, A. (2015). A Functional and Putative Physiological Role of Calcitriol in Patched1/Smoothened Interaction. The Journal of biological chemistry 290, 19614-19628.

Lu, X., Liu, S. and Kornberg, T. B. (2006). The C-terminal tail of the Hedgehog receptor Patched regulates both localization and turnover. Genes \& development 20, 2539-2551.

Marigo, V., Davey, R. A., Zuo, Y., Cunningham, J. M. and Tabin, C. J. (1996). Biochemical evidence that patched is the Hedgehog receptor. Nature 384, 176-179.

Myers, B. R., Neahring, L., Zhang, Y., Roberts, K. J. and Beachy, P. A. (2017). Rapid, direct activity assays for Smoothened reveal Hedgehog pathway regulation by membrane cholesterol and extracellular sodium. Proceedings of the National Academy of Sciences of the United States of America 114, E11141-E11150.

Porter, F. D. (2002). Malformation syndromes due to inborn errors of cholesterol synthesis. J Clin Invest 110, 715-724. 
Porter, F. D. and Herman, G. E. (2011). Malformation syndromes caused by disorders of cholesterol synthesis. J Lipid Res 52, 6-34.

Prabhu, A. V., Luu, W., Sharpe, L. J. and Brown, A. J. (2016). Cholesterol-mediated Degradation of 7-Dehydrocholesterol Reductase Switches the Balance from Cholesterol to Vitamin D Synthesis. The Journal of biological chemistry 291, 8363-8373.

Ran, F. A., Hsu, P. D., Wright, J., Agarwala, V., Scott, D. A. and Zhang, F. (2013). Genome engineering using the CRISPR-Cas9 system. Nat Protoc 8, 2281-2308.

Roberts, B., Casillas, C., Alfaro, A. C., Jägers, C. and Roelink, H. (2016). Patched1 and Patched2 inhibit Smoothened non-cell autonomously. Elife 5, e17634.

Roessler, E., Belloni, E., Gaudenz, K., Jay, P., Berta, P., Scherer, S. W., Tsui, L. C. and Muenke, M. (1996). Mutations in the human Sonic Hedgehog gene cause holoprosencephaly. Nature genetics 14, 357-360.

Sharpe, H. J., Wang, W., Hannoush, R. N. and de Sauvage, F. J. (2015). Regulation of the oncoprotein Smoothened by small molecules. Nature chemical biology 11, 246-255.

SMITH, D. W., LEMLI, L. and Opitz, J. M. (1964). A NEWLY RECOGNIZED SYNDROME OF MULTIPLE CONGENITAL ANOMALIES. J Pediatr 64, 210-217.

Sokol, J., Blanchette Mackie, J., Kruth, H. S., Dwyer, N. K., Amende, L. M., Butler, J. D., Robinson, E., Patel, S., Brady, R. O., Comly, M. E., et al. (1988). Type C Niemann-Pick disease. Lysosomal accumulation and defective intracellular mobilization of low density lipoprotein cholesterol. The Journal of biological chemistry 263, 3411-3417.

Taipale, J., Cooper, M. K., Maiti, T. and Beachy, P. A. (2002). Patched acts catalytically to suppress the activity of Smoothened. Nature 418, 892-897.

Takeyama, K., Kitanaka, S., Sato, T., Kobori, M., Yanagisawa, J. and Kato, S. (1997). 25Hydroxyvitamin D3 1alpha-hydroxylase and vitamin D synthesis. Science (New York, N.Y 277, 1827-1830.

Tint, G. S., Irons, M., Elias, E. R., Batta, A. K., Frieden, R., Chen, T. S. and Salen, G. (1994). Defective cholesterol biosynthesis associated with the Smith-Lemli-Opitz syndrome. The New England journal of medicine 330, 107-113.

Tseng, T. T., Gratwick, K. S., Kollman, J., Park, D., Nies, D. H., Goffeau, A. and Saier, M. H. J. (1999). The RND permease superfamily: an ancient, ubiquitous and diverse family that includes human disease and development proteins. Journal of molecular microbiology and biotechnology 1, 107-125.

Wichterle, H., Lieberam, I., Porter, J. A. and Jessell, T. M. (2002). Directed differentiation of embryonic stem cells into motor neurons. Cell 110, 385-397. 
bioRxiv preprint doi: https://doi.org/10.1101/2020.12.10.420588; this version posted February 9, 2021. The copyright holder for this preprint (which was not certified by peer review) is the author/funder, who has granted bioRxiv a license to display the preprint in perpetuity. It is made available under aCC-BY-NC-ND 4.0 International license.

Zhang, Y., Bulkley, D. P., Xin, Y., Roberts, K. J., Asarnow, D. E., Sharma, A., Myers, B. R., Cho, W., Cheng, Y. and Beachy, P. A. (2018). Structural Basis for Cholesterol Transport-like Activity of the Hedgehog Receptor Patched. Cell 175, 1352-1364.e14. 